\title{
LA CRISIS DEL CAMPESINADO FRENTE A LA MODERNIDAD. EL FENÓMENO DEL ÉXODO RURAL UNA APROXIMACIÓN ANTROPOLÓGICA Y LITERARIA
}

\author{
José Manuel Estévez Saá
}

\begin{abstract}
There is a group of writers and thinkers who, in one form or another, attempts to call our attention to the peasant world. This group of intellectuals portrays, in a more or less similar fashion the decline of peasant society in the modern world as observed in two important phenomena: rural depopulation and the exodus from the land.

They are sons of their own culture, and despite the geographical diversity and their different ideological colourings, they have all lent their literary voice, at the onset of the twenty-first century, to a way of life and social organisation which, if it still exists, is, without exaggeration, obsolete in relation to the advanced societies. These intellectuals clearly show, on the one hand, the smooth course of new technological and scientific advances; and on the other, the state of progressive underdevelopment and poverty to which a large part of society is subjected.

As an example, we will see how through John Berger's Pig Earth we perceive the voice of a new type of intellectual who, from his role as a writer, reflects on the bases and principles of traditional peasant society, in order to take part, in some way, in the debate concerning their possible adaptation to a modernity which is the standard of the modern world.
\end{abstract}

Dentro de la literatura actual mundial nos encontramos con una serie de intelectuales que ponen de manifiesto el curso parejo que corren los nuevos avances técnicos y científicos por un lado y, por otro, el estado de progresivo subdesarrollo y pobreza en que se va sumiendo una buena parte de la sociedad. Es precisamente dentro de esos sectores sociales más explotados y pobres donde nos encontramos con el mundo campesino que aparece como la víctima directa de ese nuevo orden generado por la modernización y que a duras penas puede mantener su identidad como seudoclase en claro peligro de extinción.

Los escritores y pensadores que, de una forma u otra, tratan de llamar nuestra atención hacia el mundo campesino, recurren a un intento reconciliador entre lo culto y lo popular, entre las nuevas formas de narración que piden prestados métodos y medios propios de otros terrenos artísticos como el cine o la propaganda, y las tradicionales técnicas de creación; tratando, en definitiva, de encontrar el método original más adecuado al éxito de su proyecto. 
Este nuevo tipo de intelectuales sabe conjugar su propio yo individual con lo más íntimo de aquellos que se encuentran a su alredor conformando el mundo socio-cultural que los adopta y arropa. El resultado creativo de esa conjunción, es una aproximación antropológico-literaria cuyo análisis nos permitirá abarcar, a un tiempo, la obra literaria de esos autores y la realidad social de la que han decidido formar parte.

El enfoque para escribir este tipo de literatura emerge de las corrientes antropológicas actuales, y supone un área de investigación interdisciplinar que se aproxima no sólo a la literatura sino también a la propia vida de las personas, considerándolas una fuente de documentación para el análisis de una cultura o un sector de la sociedad determinado (Escobedo \& Caramés 1994:60). El método de la Antropología Literaria se basa en la observación y el análisis de los grupos humanos a través de los textos. Pretende construir modelos de vida en el propio texto literario, y establecer la generalidad que define a una cultura o grupo social determinado, en nuestro caso, la sociedad rural.

El autor actual hace gala de una gran libertad que resulta evidente a la hora de mezclar sin rubor ficción y realidad, creación e historia, discurso narrativo y discurso social o filosófico, literatura y política... Se trata de un grupo de intelectuales que muestran el estado de anquilosamiento en que se encuentra el campesinado ante el mundo moderno, la nueva tecnología y el exacerbado crecimiento de las ciudades. Son hijos de su propia cultura y, pese a la diversidad geográfica y sus distintos tintes ideológicos, todos ellos prestan su voz literaria, a las puertas del siglo XXI, a una forma de vida y organización social que, si bien aún existe, resulta, como poco, obsoleta en relación a las sociedades avanzadas.

Dentro de ese grupo de escritores ${ }^{1}$, en España son varios los autores que, desde su producción temprana, se muestran portavoces del drama rural, con obras como La Familia de Pascual Duarte (1942) de Camilo José Cela (n.1916), en la que un criminal es al mismo tiempo caracterizado como azote de sus presas y víctima de la miseria, la ignorancia y la nulidad administrativa de la situación del campo; o El disputado voto del señor Cayo (1978) de Miguel Delibes (n.1920), donde se observa la exaltación de los modos de vida y de organización social del campo frente a la política del Estado.

Sin salir de España, Jesús Fernández Santos (1926-1988), plantea la inoperancia y pasividad de los campesinos ignorantes frente al caciquismo opresor en Los bravos (1954); y Jesús López Pacheco (n.1930) en Central eléctrica (1958), nos muestra la ineficacia del progreso tecnológico en la recuperación del campo. José Manuel Caballero Bonald (n.1926) en su novela Dos días de Septiembre (1961), nos describe la vida y problemas de un pueblo vinatero andaluz. La misma preocupación por el mundo rural la muestra el también andaluz, Alfonso Grosso (1928-1993), con sus novelas Un cielo dificilmente azul (1962) y La zanja (1961), ejemplo, ésta última, del estado de explotación al que el campesino andaluz se ve sometido por los caciques y el conflicto social a partir de ahí originado.

\footnotetext{
${ }^{1}$ Para un análisis exhaustivo de la literatura contemporánea, véase la obra de E. Iáñez Pareja, El Siglo XX. Literatura Contemporánea Vol.9 (Barcelona 1995) Tesys-Bosch.
} 
En cuanto a la literatura gallega contemporánea, son varios los nombres que retoman la fructífera tradición temática que se centra en los problemas del hombre del campo. Dentro de la narrativa de postguerra, nos encontramos con un Xosé Neira Vilas (n.1928), que se acerca al mundo rural denunciando la incertidumbre sociocultural en la que se encuentra el campesino, prestando atención a la problemática de la emigración en obras como Memorias dun neno labrego (1961), Cartas a Lelo (1971), o Aqueles anos do Moncho (1977). Representando a la nueva narrativa, la novela Adiós María (1971) de la poetisa Xohana Torres (n.1931), alude, al impacto que sufre el campesino a su llegada a la ciudad, desde la óptica de la urbe. También la narrativa más reciente de Manuel Rivas (n.1957), resalta la problemática del choque entre la realidad urbana, basada en la tecnología, y el mundo rural tradicional, en obras como Un millón de vacas (1990) o Os comedores de patacas (1991). De modo semejante, Suso de Toro (n.1956) o Carlos Mella (n.1930) con su novela corta Bieito, Bieito (1993), dejan constancia de los cambios traumáticos surgidos de la interpenetración de la realidad rural y urbana, mostrando -sobre todo en el caso de Mella-, a la sociedad rural como preferible y superior desde el punto de vista existencial.

En Portugal, sobresalen tres nombres: Joäo de Araújo Correia (1899-1985), Alfonso Ribeiro (n.1911) y Manuel Ferreira (n.1917). Los tres se caracterizan por una literatura capaz de recrear el devenir de una vida rural, caracterizada por el desequilibrio del campo frente a la ciudad y la incertidumbre que para el campesino supone siempre la emigración.

En lo que a la Italia actual se refiere, una serie de autores tratan de buscar nuevas formas de expresión que den una más nítida cuenta de los aspectos cambiantes de la sociedad, las costumbres y la cultura actuales, por medio de la fusión entre tradición y modernidad. Son escritores que se han sentido impulsados a reflexionar en sus obras acerca del mundo del campesino. Este es el caso de Fulvio Tomizza (n.1935), con su novela La chica de Petrovia (1963), y de otros muchos que siguen la estela de neorrealistas como Cesare Pavese (19081950) o Alberto Pincherle (n.1907), quienes fueron capaces de mostrar la Italia profunda, campesina e industrial, en obras como La luna y las hogueras (1950) o Nuevos cuentos romanos (1959), respectivamente.

También, en Alemania y, más concretamente, dentro del llamado "realismo socialista," nos encontramos a un Heiner Müller (n.1926) ampliamente asociado por la crítica actual a la creación del drama agrario y con obras como La desplazada, o la vida en el campo (Die Umsiedlerin oder Das Leben auf dem Lande, 1961); o a Erwin Strittmatter con su novela Ole Bienkopp (1963), cuestionando seriamente la preparación del campesinado ante los nuevos sistemas de explotación agrícola. Mucho más optimista y menos reacio al poder destaca Hermann Kant (n.1926), en una de cuyas novelas, El aula (1964), canta utópicamente las bondades de un régimen que por medio de un sistema universitario accesible y abierto sugiere la preparación del campesinado de cara a la asunción de tareas de responsabilidad social.

En cuanto a lo que hasta hace unos años daba en denominarse literatura soviética encontramos escritores que van desde el "reaccionario" Vladímir Tendriakov (n.1923) y Alexandr Tvardovski (1910-1971), representante de la "transición", hasta el más actual escritor siberiano Valentín Rasputín (n.1937), pasando por los "revisionistas" Alexandr 
Yashin (1913-1970) o Yuri Naguibín (n.1920), cuya crítica común a los problemas y a las condiciones actuales del campo, y a los ineficaces modelos de explotación agrícola, desemboca en una apología de los modos de vida tradicionales de los campesinos. Todos se preocupan por el problema de la tradición y la identidad cultural de los campesinos enfrentada con el ambivalente y destructivo "progreso".

Incluso en la literatura japonesa actual nos podemos encontrar con el Nobel, Kenzaburo Oé (n.1935), caracterizado por la defensa del mundo rural de la aldea y su naturaleza frente a la actual civilización urbana, causante de la progresiva perdida de la identidad de su país, gracias a obras como La presa (1958).

Siguiendo una estela pareja, en la literatura estadounidense actual descubrimos una serie de autores que representan lo que ha dado en denominarse "novela del sur." Dos buenos ejemplos los encontramos en Eudora Welty (n.1909), cuyos relatos - ej. El corazón de los Ponder y Una cortina de follaje-, recrean al Mississippi como símbolo de un modo de vida sencillo basado en el respeto a la familia, y el incondicional amor a la tierra y a las tradiciones, tan característico del ambiente rural; y en James Dickey (n.1923) en una de cuyas novelas, Deliverance (1970), la vida rural es ensalzada en detrimento de la vida urbana de la capital.

Todos estos escritores pertenecen a un conjunto de intelectuales que en un momento dado de sus vidas deciden formar parte de esas bolsas de resistencia que se crean ante las injusticias y desigualdades que asolan al campesinado y que son fruto del nuevo orden mundial, supuestamente basado en el progreso y la modernización. Todos ellos tratan de buscar soluciones o, al menos, mostrar el estado de una cuestión ante la que no se muestran indiferentes. Para ello, algunos como Miguel Delibes, James Dickey o Kenzaburo Oé, mostrarán las virtudes y los valores del mundo rural; otros como Hermann Kant, recrearán un mundo utópico en el que el campesino logra su plena integración social; y muchos, como Alfonso Grosso, Cela o Yuri Naguibín, asumirán un punto de vista mucho más crítico, denunciando el desamparo del mundo del campo ante la mirada inoperante de los que ostentan el poder.

En esta misma línea generacional de compromiso con el campesinado y en representación de las letras inglesas actuales, nos encontramos con el escritor británico autoexiliado en Francia, John Berger (n. Londres, 1926), y su trilogía Into Their Labours (1992). La trilogía consta de un primer volumen, Pig Earth (1979), en el que a través de una serie de relatos, poemas y ensayos, Berger muestra el modo de vida tradicional de un pueblo de montaña en donde un grupo de campesinos dedicados a sus tareas permanecen, a la vez, ajenos a la historia, aislados del mundo exterior, y temerosos de un futuro cada vez más incierto basado en el progreso que los fuerza a una desesperada emigración. El segundo volumen, Once in Europa (1983), profundiza un poco más en la modernización de ese mundo campesino. Una serie de historias en las que el amor y el desamor constituyen una parte esencial en la vida de los personajes muestran cómo ese mundo campesino descrito en el primer volumen tiene que enfrentarse paulatinamente a un proceso de desarrollo y modernización que genera constantes injusticias. El tercer volumen, Lilac and Flag (1990), nos presenta a estos y otros muchos personajes en un ambiente bastante 
distinto, la metrópoli. Aquí, estos campesinos exiliados se encuentran como perdidos dentro de un ambiente y unas costumbres que les son ajenas. Es por ello que éstos se sienten miserables en un mundo marcado por el crimen y la mezquindad de los patronos, y añoran constantemente su vida rural dejada atrás.

La recreación de la realidad campesina basada en una inevitable y forzosa necesidad de emigrar presente en el primer volumen de la trilogía constituirá, ahora, el objetivo de mi mirada.

En su obra Pig Earth ${ }^{2}$ (1979), John Berger nos demuestra que es capaz de combinar relatos, poemas y ensayos de pareja intensidad sociológica y literaria. Ocho historias de extensión variada ("A Question of Place", "A Calf Remembered", "The Great Whiteness", "An Independent Woman", "The Wind Howls Too", "Addressed to Survivors", "The Value of Money", "The Three Lives of Lucie Cabrol") se alternan con otros tantos poemas (Death of La Nan M., Ladle, Easter, Ladder, Village Maternity, Sunset, Hay, Potatoes), una narración de carácter ensayístico (“An Explanation”), y un ensayo sociopolítico ("Historical Afterword").

Esta original técnica narrativa que acepta la libre combinación de relato, poesía y ensayo, emerge tanto del debilitamiento de normas estrictas, como de la tensión entre determinadas formas tradicionales y los nuevos modos de creación literaria, tan característica de un momento histórico en el que tanto se habla de la presunta crisis de los géneros en general y de la novela en particular. El equilibrio resultante revela la vitalidad de la cultura literaria que caracteriza las últimas décadas de este siglo y que John Berger consigue manifestar no sólo en Pig Earth y su trilogía Into Their Labours, sino también en el resto de su producción literaria.

Pese a ello, el análisis formal del estilo bergeriano no constituirá en sí mismo un objetivo primordial en el desarrollo de nuestro ensayo. Será el aspecto social al que alude el texto y no el formal, el fondo y no la forma, lo que entrará dentro del punto de mira de nuestro estudio actual. Será en el Berger pensador, provocador y armador de ideas en el que centraremos nuestra mirada, en detrimento momentáneo del Berger analista y artesano de la palabra.

$\mathrm{Y}$ es que dentro de esa realidad social que Berger pretende recrear, nos encontramos con un tema fundamental y constante a lo largo de todas y cada una de las historias de que se compone el libro: la emigración y el éxodo rural. La relevancia del tema, que justifica la atención especial que le dedicaremos, se acentúa si tenemos en cuenta tres factores principales. En primer lugar, el hecho de que también en torno a él, desarrollará John Berger los dos libros posteriores que completarán su trilogía Into Their Labours. En segundo lugar, por constituir éste, para John Berger, uno de los fenómenos más importantes de los últimos tiempos. Así lo pone de manifiesto Berger en una entrevista concedida a

\footnotetext{
${ }^{2}$ Las alusiones y citas pertenecientes a Pig Earth irán paginadas con su simple numeración entre paréntesis, y corresponderán a la primera edición de Pig Earth, publicada en Londres por la editorial Writers and Readers en 1979.
} 
Jorge Riechmann: "Éste es sin duda uno de los fenómenos más importantes del siglo, y ha influido en mi trabajo" (Riechmann 1995:18). En tercer lugar, por el hecho de que el propio Berger -como él mismo afirma en la entrevista- ha vivido en su propia familia la experiencia de la emigración, de ahí su especial sensibilidad hacia el tema:

Un abuelo mío -el padre de mi padre- fue un emigrante de Trieste: es decir, yo mismo provengo de una familia de emigrantes. Cuando tenía dieciséis años dejé de estudiar y marché a Londres. En muy poco tiempo mi círculo de amigos se componía casi exclusivamente de emigrantes. Con ellos me sentía como en casa... (Riechmann 1995:18)

A través de una serie de personajes y de situaciones concretas, Berger demuestra un alto nivel de conciencia social revelando las condiciones sociales y los sentimientos de un sector de la sociedad en particular. El campesino se ve obligado a abandonar su casa y sus tierras hasta llegar a depender de una forma excesiva de las ciudades. Y es sobre esta trágica realidad (recordemos que el campesino considera su lugar de nacimiento como el centro de su mundo (11)), sobre la que Berger realiza una completa y dinámica reflexión. Berger es capaz de dirimir tanto las causas que hacen inevitable las migraciones, como las notables diferencias existentes entre el mundo urbano y el mundo rural, exponiendo, incluso, el papel discriminatorio y secundario que desempeñarán los emigrantes en sus nuevos destinos, gracias, todo ello, a un lenguaje preciso y directo.

John Berger reflexiona sobre el hecho de que cada vez es mayor el numero de personas que dejan de trabajar sus tierras para dedicarse a otros trabajos que puedan ser mejor remunerados y de mayor prestigio social. Con todo, muchas de estas personas no se ven obligadas a abandonar sus lugares de residencia originarios en pueblos y aldeas, debido, entre otros, a una serie de factores tales como: a la creación de industrias generadoras de trabajo cada vez más alejadas de la ciudad y por tanto más cerca de los centros rurales, o al desarrollo de los medios de transporte (motorización y transporte público), que permite que muchas personas residan en las zonas rurales al tiempo que trabajan en las ciudades. Es por estas y otras causas por lo que cada vez se habla más del distanciamiento entre agricultura y pueblo, o en palabras de Corrado Barberis, "le divorce entre l'agriculture et la campagne". Así, Barberis afirma que

l'exode agricole, c'est à dire l'abandon du secteur primaire, est généralement plus élevé que l'exode rural, pris dans son sens d'abandon d'une zone géographique. (Van Nieuwenhuijze 1972:17)

Según un temprano estudio realizado sobre Francia -y elegimos Francia por constituir el escenario en el que se ambienta Pig Earth-, se llegó a la conclusión de que sobre una población de 225.000 hombres que han abandonado la agricultura de 1959 a 1964, un 60\% no ha emigrado, y únicamente un $10 \%$ se ha visto obligado a cambiar de región, de modo que casi un $78 \%$ de los que se desplazan permanecen en comunidades rurales (Praderie \& Passagez 1966:30). Este tipo de éxodo diario es el realizado por ejemplo por Edouard en "The Value of Money", que se ve obligado a desplazarse a distintas localidades para desarrollar su trabajo de vendedor, pero que diariamente vuelve a casa donde reside con otros miembros de su familia. 
El éxodo rural se está reduciendo en perjuicio del éxodo agrícola. Esta última frase mal entendida podría producir una sensación de falsa contradicción u oposición a todo lo que hasta aquí llevamos dicho. Si bien para el desarrollo del presente apartado constantemente estamos refiriéndonos al concepto de "éxodo rural" como la despoblación cada vez más acentuada de pueblos y campos en general, en este momento se exige hacer un tratamiento del concepto con una mayor precisión, de modo que nos resulte más fácil distinguir entre lo que se ha dado en llamar "éxodo agrícola" y "éxodo rural". Entenderemos por "éxodo agrícola" el constante y acelerado abandono del trabajo de la tierra, y los campos de cultivo y derivados, del mismo modo que entenderemos por "éxodo rural" el abandono de pueblos y aldeas como lugar de residencia por parte de la población. En Pig Earth, sin embargo, y pese a esporádicas vivencias de este tipo de éxodo agrícola, como la señalada anteriormente, es el otro tipo de éxodo, aquel en el que abandono de campo y pueblo están relacionados, el que es recreado por John Berger de una manera más precisa a lo largo de cada una de las historias en las que el problema del éxodo y la emigración aparecen planteados. Tanto a la hora de indagar las causas y consecuencias del éxodo, como de hacer un análisis comparativo del pueblo y la ciudad, de realizar una valoración del fuerte impacto que sufren los emigrantes cuando llegan por primera vez a la ciudad, o de buscar posibles soluciones, será precisamente a ese concepto más genérico de éxodo, en el que va incluido abandono de tierras y pueblo o lugar de residencia, al que estaremos haciendo alusión cuando nos refiramos al término de éxodo rural o emigración. Señalar, por tanto, que en el desarrollo de este ensayo, "éxodo rural" y "emigración" serán entendidos como sinónimos.

Quizá sea aconsejable, si tratamos de analizar un problema de la complejidad del éxodo y la emigración, hacerlo, como el propio Berger propone, desde un punto de vista genérico tratando de buscar las causas que simplemente lo hacen inevitable, aunque a partir de las situaciones y los personajes concretos de cada una de las historias de que se compone el libro.

John Berger nos presenta el éxodo como un fenómeno que trasciende a cualquier nación o región del planeta en particular, y que tiene diversas y profundas raíces de carácter cultural, económico, social y político:

En nuestro siglo, muchos más millones de personas se han visto obligadas a abandonar su lugar natal y emigrar -por razones económicas y políticas- que en ninguna época anterior. (Berger en Riechmann 1985:18).

El fenómeno del éxodo es un evidente ejemplo del tremendo cambio socioeconómico que esta teniendo lugar en todo el mundo; y cuyas consecuencias, como manifiestan María Carmen Faus-Pujol y Antonio Higueras-Arnal, son impredecibles:

Rural depopulation is probably one of the most reliable indicators of the deep socioeconomic change which is taking place in the world, and whose final result cannot be predicted. (1986:10) 
Constituye precisamente el éxodo uno de los factores decisivos que motivan la constante desaparición del campesinado. Berger explica que en Francia, en la actualidad, el número de campesinos que abandonan el campo cada año es de 150.000. Y asegura que, según los planificadores económicos de la $\mathrm{CEE}$, la previsión de desaparición del campesinado se sitúa en un período de tiempo de no más de un siglo o quizá menos (209). También advierte John Berger que el campesino está empezando a depender tanto de las ciudades que su existencia como grupo está por desaparecer:

Soon the world countryside may be dependent on the cities even for the food its own rural population requires. When and if this happens, peasants will have ceased to exist. (211)

El movimiento de masas a lo largo de un período demasiado extenso de la historia ha sido del campo a la ciudad o de estancadas áreas urbanas hacia zonas más desarrolladas. Este éxodo masivo afecta tanto a naciones en proceso de gran desarrollo a todos los niveles (Estados Unidos, Inglaterra, Italia, o Francia, como veíamos anteriormente), como a los países socialistas de la Europa del Centro en los que este fenómeno está muy generalizado. John Berger, refiriéndose a los países menos desarrollados hace un retrato de algunas de las causas que motivan el éxodo, como los equivocados sistemas de tenencia de tierra, la imposición de monocultivos, la marginación de las granjas de subsistencia, etc.:

[...] the systems of land tenure [(...)], the imposition of monocultures for the benefit of corporate capitalism, the marginalisation of subsistence farming, and, only because of these other factors, the mounting population, cause more and more peasants to be reduced to such a degree of absolute poverty that, without land or seed or hope, they lose all previous social identity. Many of these ex-peasants make for the cities [...]. (210)

Los resultados de este éxodo masivo al que alude Berger son alarmantes. Así, Rogers, atendiendo al fenómeno del éxodo rural como el primer factor determinante, llama la atención sobre el desproporcionado crecimiento de la población urbana durante los últimos tiempos:

In 1800 , there were about 25 million people living in urban areas. In 1980, there were about 1.8 billion. By the year 2000, it is estimated that this number will rise to 3.2 billion, a 128fold increase in two centuries. (Rogers \& W. 1982 en Bogin 1988:90)

Barry Bogin, del departamento de Ciencias del Comportamiento de la Universidad de Michigan, afirma que la población que emigra del campo a la ciudad la constituyen principalmente personas con mejor nivel educativo que las que deciden quedarse en las zonas rurales, de un estatus socioeconómico ligeramente más alto, más jóvenes, y menos conservadores en lo que a valores tradicionales culturales se refiere (Mascie-Taylor \& Lasker 1988). En otras ocasiones, sin embargo, es la necesidad absoluta que impide la supervivencia en según qué zonas rurales la que motiva la emigración.

Cada una de estas características aparece recreada por John Berger conformando perfectamente el retrato de aquellos que en las historias de Pig Earth se ven obligados de alguna manera a tomar la decisión de abandonar sus casas en busca de trabajo y desarrollo personal. Esto lo podemos observar con facilidad en el personaje principal de una de las 
historias de Pig Earth, "An Independent Woman", de nombre Catherine, y en su hermano Mathieu, quienes se ven obligados a viajar a París, a ganar su primer sueldo. La propia Catherine llega incluso a quejarse de que:

[...] each year of her life more men had left the valley, and she herself had inherited too little to propose to any of them that they remain.(30)

También el joven Émile, hermano de la Cocadrille en "The Three Lives of Lucie Cabrol", con dieciséis años abandona su casa para ir a París y ponerse a trabajar de fogonero; o Jean, el personaje narrador de esta historia, que también se vio obligado a emigrar a Argentina; o Pépé en "The Wind Howls Too", quien con quince años siente la necesidad de ir a París en busca de trabajo al ver que "the family could not live off four cows" (45). Del mismo modo, en "The Value of Money", los dos hijos de Marcel deciden dejar sus casas para ir a las grandes poblaciones a ganar dinero: Michel trabajando en una fábrica, y Edouard como representante o vendedor ambulante (74).

Junto con estos, otros muchos personajes también deciden un día lanzarse a la hazaña de la emigración, aventurarse por ciudades y países desconocidos en busca de algún beneficio -casi siempre sin saber muy bien de qué tipo-, como única alternativa al estado de circunstancias adversas en que se encuentran. Muchas veces esa misma emigración, en un principio deseada, promete cosas que no se cumplen una vez alcanzada. Así lo manifiesta Jean en "The Three Lives of Lucie Cabrol":

On feast days in the Argentine the peones killed and ate turkeys: emigration offered me no new promises. The promise of the Place d'Étoile and the promise of the Arenne Corrientes in Buenos Aires were simply revivals of what I had already hoped in the village. I couldn't have imagined those places from the village, yet I did imagine my pleasure, the same pleasure they promised and didn't give me. (186)

Quizá sea por esta cruda y frecuente realidad de las promesas incumplidas, por las desilusiones y la tristeza resultante de un esfuerzo vano, por lo que la emigración siempre haya sido considerada como algo que merece tanto respeto desde el punto de vista de las grandes decisiones que el hombre tantas veces debe tomar si pretende, de alguna forma, cambiar el transcurso de su vida. En este sentido resultan memorables las palabras pronunciadas en 1954 por Ramón Otero Pedrayo:

Quisiéramos acercarnos a la emigración, con el respeto y el amor debidos a toda forma de vida en que el dolor y la esperanza combinan en variados y renovados tejidos la trama de las horas y los días, en que no permanece imperturbada ni la más leve fibra del ser y el carácter. Pocos emigrantes, quizá en años enteros ninguno, pueden evaluar la variedad y alteza de las eviternas esencias perturbadas, de los númenes desvelados por el simple hecho de partir [...]. La cultura geórgica, el saber de tradición y secular esperanza para el emigrante declara su intimidad. Es el partir poner a la luz del día lo oculto y esencial de la flor única de la vida y la estirpe. (Otero Pedrayo 1954:13)

Las pestes y las hambrunas también motivaron que a lo largo de la historia los campesinos, como señala Berger en el "Historical Afterword": "were forced to abandon the village and become wage earners" (200). 
Un personaje, Marcel, en “The Value of Money" ve cómo su hijo Edouard pretende vender la prensa del lagar como representación de todo lo que supone el mundo tradicional campesino que apenas da ya beneficios, y se lamenta de que cada vez sean más los que se están apartando de la tierra: "The world has left the earth behind it, said the father" (86). Edouard, un poco más adelante, responde a las críticas de su padre diciendo:

Half the men here had to emigrate because there wasn't enough to eat! Half the children died before they grew up! Why don't you admit it? (86)

En "The Three Lives of Lucie Cabrol", Jean tiene que abandonar el pueblo y marcharse a París y luego a Argentina. La vida de escasez y necesidad que causa su afán por adquirir riquezas hace que recuerde la contestación de la Cocadrille cuando, ante la pregunta "Is love of the good things of life ever justified?", ésta le responde "Yes, there is a justified love of the good things of life and this love inspires foresight and thrift" (186). El mismo Jean, en un momento dado, trata de buscar las razones del éxodo generalizado que afecta a su pueblo y se pregunta "Why were so many of us obliged to go to Paris as chimney sweeps?" (187).

Berger en el "Historical Afterword" nos da alguna de las claves del éxodo, al explicar por qué desde tiempo atrás, los pocos campesinos que continuaban trabajando (a minority) eran considerados una especie de supervivientes (survivors), dado que la mayoría "died young, emigrated or became paupers" (199).

Las mejoras técnicas llegaron a suponer, ya desde el siglo XVIII, una amenaza para el mundo campesino, que se vio, también de este modo, obligado a abandonar sus casas y a emigrar en busca de trabajo, puesto que los nuevos sistemas agrícolas y la incorporación de maquinaria, redujeron el número de brazos necesarios para realizar las labores de un campo cada vez más mermado:

[...] the large-scale commercialisation and colonialisation of agriculture, the inadequacy of ever smaller plots of land to support entire families, hence large-scale emigration to the cities where the sons and daughters of peasants were absorbed into another class. (200)

Con todo, algunos se aprovecharon de ese nuevo estado de circunstancias, para también dejar de ser campesinos, años más tarde:

One should add that under these new conditions a few peasants became rich, but in doing so they also ceased, within a generation or two, to be peasants. (200)

La mayoría de las veces, estos campesinos emigrantes no pueden llevarse consigo a sus familias, produciéndose, los resultados negativos que esto acarrea, como vemos en las palabras de André en "The Wind Howls Too", quien nos explica la habitual y frecuente dureza de los inviernos para los ancianos, las mujeres y, sobre todo, los niños que se quedan privados de la figura paterna:

Those who stayed behind were the women, the old and the young. During the winter the fact that I had no father was scarcely remarkable; half the children of my year were temporarily without fathers. (43) 
Vemos, pues, que si los motivos que impulsan al hombre del campo a emigrar son múltiples y variados, entre todas las causas, María Carmen Faus-Pujol sugiere, sin embargo, un denominador común que pese a que pueda estar algo alejado de una concepción marxista, merece la pena que quede aquí constancia del mismo como un punto de vista más, y que es, según ella, la aspiración que todos tenemos de mejorar nuestras condiciones de vida: "the universal aspiration, and right, which we all have to improve our living conditions" (1986:6).

En líneas generales, podemos decir que toda mejora, en lo que a condiciones de vida se refiere, lleva asociada una forma de movilidad social. Cuando se trata de zonas tradicionalmente rurales, la movilidad social es menor, debido a que normalmente se ve frenada por toda una serie de rígidas estructuras socioeconómicas dominantes, y es por ello que la única forma de mejorar las condiciones de vida y romper con todas esas ataduras socioeconómicas es emigrar. Como también decíamos anteriormente, la emigración se lleva a cabo desde las zonas rurales hacia zonas urbanas (ciudades), en las que, al menos a priori, la movilidad es mayor y resulta más fácil conseguir la promoción social y la mejora en las condiciones de vida.

En este caso, la visión del emigrante como una "víctima de las circunstancias" se ve sustituida por una visión más positiva basada en valores cercanos a la autoestima, a la propia dignidad humana. En las zonas rurales, las personas suelen tener más dificultades a la hora de conseguir tanto la ansiada riqueza como muchos de los bienes culturales. Por tanto, parece lógico que estas personas acaben por trasladarse a otros lugares donde puedan promocionarse y cultivar, al menos en teoría, los distintos aspectos de su personalidad.

Esto no es, sin embargo, tan sencillo. En la decisión de emigrar que toman los personajes de Berger, la presunción de mejora en la calidad de vida va implícita, como podemos inferir, por ejemplo, de las palabras de la Cocadrille en "The Three Lives of Lucie Cabrol": "there is a justified love of the good things of life" (186); o de Jean, en el mismo relato: "I did imagine my pleasure, the same pleasure they promised and didn't give me" (186). Faus-Pujol llama la atención sobre lo que una noción como la de "calidad de vida" (quality of life) implica. Ella señala que se trata de una noción subjetiva y que puede llevar a errores al poder llegar a confundirla con la de "nivel de vida" (standard of living). Explica esta autora que, de alguna manera, la noción de nivel de vida está relacionada con la posición desempeñada en la escala social, entre otras cosas, porque la evaluación que las personas hacemos del nivel de vida se basa en indicadores puramente materiales que nosotros, de forma consciente o no, hemos incorporado a nuestro sistema de valores y que, desde ese mismo instante, se ha convertido en un sistema materialista. Faus-Pujol señala que lo que el emigrante siente una vez asentado en una zona geográfica nueva es que está en posición de adquirir una calidad de vida nueva a pesar de que, en la mayoría de los casos, no llega a conseguirlo, a no ser a través de indicativos materiales.

Para resumir todo lo que a partir de Pig Earth hemos dicho respecto a las causas del éxodo rural, las palabras de Su Santidad el Papa Juan XXIII en su Encíclica "Mater et Magistra" son fundamentales: 
The exodus from the land to other sectors of production is often due to reasons of economic development. But it is undeniable that the exodus from the land is due to the fact that the agricultural sector everywhere is depressed, both in terms of productivity, and in terms of standard of living of the rural population. (Faus-Pujol 1986:19)

En lo que concierne a la vida en las ciudades de destino de esos emigrados en contraposición con los pueblos que han dejado atrás, hemos de decir que éstas constituyen un mundo, la mayoría de las veces, antagónico.

En Pig Earth, la sensación de aislamiento y soledad que experimenta el campesino cuando llega a la ciudad aparece descrita de forma muy explícita. En "The Three Lives of Lucie Cabrol", Jean reflexiona acerca de este tipo de sensaciones adquiridas. En el campo él es consciente de las labores que lleva a cabo así como de la función que realiza, teniendo una grata sensación de control y responsabilidad sobre todo aquello que acontece a su alrededor. Por el contrario, una vez que llega a la ciudad, observa que todo lo que allí sucede está fuera de su control. Se ve aislado, expectante, como quien asiste a algún tipo de ritual que se desarrolla detrás de un vidrio infranqueable a la vez que transparente. Y así, en la página 150, el "yo" protagonista, que en este caso resulta ser el propio Jean, manifiesta:

When you arrive in the city, where so much is happening and so much is being done and shifted, you realise with astonishment that nothing is in your control. (150)

Jean no se siente capaz de franquear esa barrera que lo excluye y le impide llegar a integrarse en el devenir del proceso de vida que engrana el conjunto de la ciudad:

It is like being a bee against a window pane. You see the events, the colours, the lights, yet something, which you can't see, separates you. (150)

Esta sensación que muestra Jean se podría explicar en términos de alienación dentro de la propia alienación que implica, de algún modo, la emigración a las ciudades. El propio Jean intenta dar explicación a lo que en esos instantes le sucede:

With the peasant it is the forced suspension of his habit of handling and doing. (150)

Llega incluso a la ironía en sus explicaciones: "That's why his hands [las del campesino, se entiende] dangle out of his cuffs so stupidly" (150).

Faus-Pujol describe las ciudades como núcleos residenciales y administrativos en los que no existe posibilidad de cohabitación, es decir, de llevar una vida auténticamente en sociedad. En la ciudad la gente vive, trabaja y ve su tiempo libre completamente saturado por distintas ocupaciones, sin llegar a cohabitar, sin sentirse parte integrante de una comunidad. Para Faus-Pujol resulta una paradoja que sea precisamente en estos sitios de carácter tan individualista ("fierce individualism") donde se concentren la mayoría de los recursos económicos, profesionales y culturales, que en teoría tendrían que contribuir, precisamente, a todo lo contrario. El pueblo, entendido como pequeña comunidad rural y por el contrario, resulta ser primordialmente un núcleo de cohabitación: 
the village lacks any other specific functions; it hardly has any contact with other nearby or faraway nuclei. (Faus-pujol \& Higueras-Arnal 1986:14)

Jean, que pese a hablar del campesino en forma impersonal, está refiriéndose a su propia experiencia como tal, da las claves que le han ayudado a él a sobrellevar esa sensación de soledad tan grande que le aporta la ciudad, en contraposición con el campo en el que lo normal es el contacto y la apertura constante hacia los demás. Entiende lo que en la ciudad acontece como una especie de representación teatral, una especie de ilusión, y continúa insistiendo en la necesidad de recurrir al silencio del secretismo íntimo y personal si se quiere lograr la superación de ese estado de circunstancias en el que tiene que sobrevivir:

You have to understand that everything you watch in the city is as unimportant as a game. Everything which impresses you about the city is an illusion. It is not easy. To be impressed and unimpressed at the same time! What really happens in a city is hidden. If you want to achieve anything it must be arranged in secret. (151)

El camino parejo que parecen seguir el constante avance de las ciudades en medios, ofertas y servicios por un lado, y el aislamiento al que tiene que estar sometido el ciudadano en estos grandes centros urbanos por otro, motivan, como manifiesta John Berger, que últimamente se esté dando un fenómeno singular en el hombre de ciudad, la progresiva atracción que siente hacia el campo como lugar de evasión:

[...] the degree and efficacy of such everyday protection has correspondingly increased. Recently the insulation of the citizen has become so total that it has become suffocating. $\mathrm{He}$ lives alone in a serviced limbo -hence his newly-awakened, but necessarily naïve, interest in the countryside. (206)

John Berger, en el "Historical Afterword" de Pig Earth, también reflexiona sobre este contraste existente entre la ciudad y el pueblo, esta vez desde una óptica diferente a la de la simple convivencia o cohabitación. Desde el punto de vista material de la actividad laboral y el estilo de vida que a diario define al campesino, la ciudad aporta, como pudimos ver, una serie de comodidades que se convierten rápidamente en una sensación de protección distinta de la que éste siente en el campo. En el campo, el campesino pese a lograr predecir algunos de los cambios que observa a su alrededor, a veces no se ve capaz de luchar contra toda una serie de factores, especialmente naturales, que hacen que su trabajo se vea, por momentos y dependiendo de distintas circunstancias ajenas a su propia voluntad, seriamente amenazado. Estas amenazas a las que el campesino ha de enfrentarse diariamente en el campo motivan esa particular sensación de falta de protección:

By contrast [con la ciudad] the peasant is unprotected. Each day a peasant experiences more change more closely than any other class. Some of these changes, like those of the seasons or like the process of ageing and failing energy, are foreseeable; many -like the weather from one day to the next, like a cow choking to death on a potato, like lightning, like rains which come too early or too late, like fog that kills the blossom, like the continually evolving demands of those who extract the surplus, like an epidemic, like locusts- are unpredictable. (206) 
Las ciudades, según explica también Berger en el "Historical Afterword", son capaces de suplir y cubrir toda una serie de incomodidades relativas o características del campo, e incluso de acentuar en gran medida esa sensación de protección que tanto añora el campesino, pese a la sensación de aislamiento que en ellas éste experimenta:

There was heating to counteract changes of temperature, lighting to lessen the difference between night and day, transport to reduce distances, [...]. There were walls and other defences against attack, there was effective law, there were almshouses and charities for the sick and aged, there were libraries of permanent written knowledge, there was a wide range of services -from bakers and butchers through mechanics and builders to doctors and surgeons- to be called upon whenever a need threatened to disrupt the customary flow of life, [...]. (206)

Reparemos ahora en la función que el campesino desempeñará una vez llegado a la urbe.

El campesino experimenta un shock cuando llega por primera vez a la ciudad. Una vez superada la decisión de desatar los lazos que le unen física y sentimentalmente a su familia y a su lugar de origen, el campesino tendrá que hacer frente a toda una serie de novedades que unas veces le endurecerán como persona y otras terminaran por desalentarlo. Cuanto mayor sea la edad del emigrante, mucho más graves serán sus problemas de adaptación a un nuevo destino asentado sobre un sistema socioeconómico, cultural e incluso lingüístico distinto. Cuando se es joven, las vigas que conforman la personalidad de cada uno se encuentran mucho menos marcadas, por lo que el shock producido por la toma de contacto con ese nuevo mundo siempre será mucho menor.

El campesino tendrá que enfrentarse a ocupaciones de distinta índole, que le supondrán esfuerzos también variados. Un estudio efectuado en 1980 por COPLAMAR (Coordinación General del Plan Nacional de Zonas Deprimidas y Grupos Marginados), demostró cómo, sólo en Méjico -una de las grandes zonas afectadas por el éxodo rural-, tres millones de personas de origen campesino disfrazan su desocupación dedicándose al comercio ambulante -lustrar zapatos, vender baratijas, "lanzar llamas"- en las esquinas de la capital (García Canclini 1982).

Así, en la ciudad los campesinos serán los que ocupen los puestos de trabajo más humildes. John Berger llama la atención sobre este hecho dedicándole una atención amplia y reflexiva.

A lo largo del libro distintos personajes se ven en la necesidad de abandonar su trabajo en el campo para realizar tareas propias de los sectores más bajos de la sociedad en las ciudades a las que se desplazan, "Many of the men went from the village to Paris to earn wages as stokers and porters and chimney-sweeps" (43). Lo comprobamos con Catherine, que trabajará de camarera en un restaurante junto a la Gare de Lyon primero, y como criada en casa de un médico luego, donde tendrá como obligaciones limpiar chimeneas, fregar los suelos o lavar la ropa. Matheu, en "An Independent Woman", también abandonará su pueblo para trabajar de portero en la ciudad. Pépé, en "The Wind Howls Too", trabajará de jornalero en las obras del Grand Palais. En "The Value of Money", Michel se empleará en 
una fábrica, Edouard realizará tareas de vendedor ambulante por las calles de la ciudad, Émile trabajará de fogonero, la Cocadrílle venderá setas y violetas, etc. Y la realidad es que, de todos los que se ven obligados a migrar, tan sólo Jean adquirirá un cierto nivel económico, que no social, cuando, tras trabajar en París de deshollinador y pasar veinticinco años en Argentina realizando distintas tareas, se desplazará hasta Montreal en donde "[...] for a while, I was rich. I had my own bar there" (135), para, luego, volver a caer en la ruina tras un inesperado incendio. En ese momento, un trabajo como vigilante nocturno le ayudará a conseguir dinero suficiente para hacerse con el billete que lo lleve de vuelta a casa.

En Pig Earth, Berger nos describe al campesino como un extraño en la ciudad, pero como un extraño que actuará con honor y dignidad en toda función que en ella realice. El campesino es un ser trabajador y responsable, con una elevada capacidad de sacrificio que le conduce a llevar a cabo cada una de las tareas que se propone de manera eficiente. Pero esto es algo que no siempre puede demostrar, debido a la escasez de oportunidades, a la falta de trabajo, a la exclusión que le impone el progreso. Pese a todo, el campesino emigrado no es un ser impaciente, sabe permanecer expectante mientras aguarda a que las cosas vayan mejorando paulatinamente:

Many of these ex-peasants make for the cities where they form a millionfold mass such as has never existed before, a mass of static vagrants, a mass of unemployed attendants: attendants in the sense that they wait in the shanty towns, cut off from the past, excluded from the benefits of progress, abandoned by tradition, serving nothing. (210)

Ramón Otero Pedrayo describe brillantemente al campesino en la emigración cuando afirma:

El emigrante ensaya mil ocupaciones. Las formas transitorias son también prueba y gusto de la libertad en los confusos suburbios, en el trato variado de gentes... Las condiciones varían. Casi indiferente al clima geográfico se siente a gusto solo... Se encuentra el hombre dueño de una fuerza segura y tranquila que pasa de un trabajo a otro trabajo, cumpliendo bien en todos y ausente de ellos, proyectado en una dirección tal vez no clara y definitiva, esperando... (Otero Pedrayo 1954:50)

Por otro lado, John Berger manifiesta en "An Explanation", la dificultad que tiene el campesino a la hora de dejar atrás todo lo que el mundo del que procede supone y adaptarse a su nueva situación como emigrado. Berger llama nuestra atención acerca del hecho de que pese a que el campesino pueda estar interesado en el mundo allende las lindes de su pueblo, es extraño que un campesino pueda trasladarse de un sitio a otro sin dejar de ser campesino:

Peasants are often interested in the world beyond the village. Yet it is very rare for a peasant to remain a peasant and be able to move. (11)

En su nuevo destino, el campesino experimenta una sensación de extrañeza que no pasa desapercibida para el resto de la población de la gran urbe. La gente de ciudad observa al campesino como forastero, como un extraño al que cada vez está más acostumbrada a ver.

También el propio campesino es consciente de las diferencias que lo definen con 
respecto al resto de los personajes de la urbe. Esta doble realidad queda sugerida por el narrador en "The Three Lives of Lucie Cabrol" cuando, al describir a la Cocadrille en la ciudad, nos dice:

She had the appearance of a pauper and at the same time she looked wilful. Such a combination in a city suggests madness. [...] This was the only moment of the week when she sat in company, although she spoke to no one except the waitress. [...] She studied the other customers, nearly all of whom were women out shopping. She noticed their hands, their made-up faces, their jewelry, their shoes with high heels. She had no wish to speak to them and she did not envy them, yet the sight of them gave her pleasure. They were a weekly proof of the extent of what money can do. (156)

El narrador utiliza el calificativo de 'locura' (madness) al referirse al aspecto que luce la Cocadrille, calificativo que más bien habrá de entenderse como "extrañeza". Esta extrañeza en el aspecto que actúa como elemento diferenciador se hace explícita a lo largo de la historia, así como a partir de la descripción del aspecto de las mujeres de la cafetería en la que, según el narrador, repara la Cocadrille -quien sin embargo no aplica el término peyorativo de locura al aspecto ostentoso y recargado de las mujeres.

El paso del tiempo contribuye a que estas diferencias de aspecto, de vestimenta, de comportamientos y costumbres se vayan limando poco a poco -otra cosa bien distinta y mucho más difícil es que éstas terminen por desaparecer. El campesino termina por acostumbrarse e intenta adaptarse a una nueva forma de hábito y vida que, en la mayoría de los casos dista mucho de aquel modo de vida propio del lugar del que procede.

El proceso de adaptación, tanto en los modos como en las formas, irá asociado a la incursión paulatina del campesino en el mundo del "materialismo desenfrenado", tan característico de la sociedad urbana. En referencia a la atracción que ese materialismo ejerce, quizá debamos recordar el tono irónico de los pensamientos que el narrador omnisciente de "The Three Lives of Lucie Cabrol" pone en la mente de la Cocadrille en el momento en que ésta observa, desde la distancia, el aspecto ostentoso de los vestidos, los maquillajes y las joyas que lucen las mujeres de ciudad que tiene ante sus ojos en la cafetería: "They were a weekly proof of the extent of what money can do" (156).

Llegados a este punto, y tras habernos planteado las causas que parecen hacer cada vez más inevitable el fenómeno del éxodo, tras estudiar el contraste existente entre la forma de vida de la ciudad y la del pueblo, y una vez realizado el análisis del shock que sufre el campesino cuando llega a la ciudad, John Berger parece dejar un final abierto al problema al no aportar explícitamente en el desarrollo de sus historias las posibles soluciones que contribuyan a erradicar o al menos a frenar tanto el fenómeno del éxodo en sí, como la posible desaparición del campesinado. Tampoco en su defecto nos ofrece Berger evidentes razones por las que considera que debiera permanecer. Así, si la tarea de trazar el cuerpo de las causas del éxodo en Pig Earth no parece fácil, mucho menos sencillo, incluso osado, resulta buscar algún tipo de solución al problema.

Sin embargo y acudiendo a los silencios textuales, podemos intuir una serie de razones no explícitas como son todo ese cúmulo de valores -no absolutos y probablemente en sí 
mismos enjuiciables-, que representa el mundo campesino, tales como el respeto a la tradición, el sentido de justicia, el esfuerzo de su trabajo incondicional, el cariño a la tierra y a la naturaleza, su sentido de la familia, etc.

Del mismo modo, pese a que en su análisis del fenómeno del éxodo Berger no hace referencia a estrategias macroeconómicas ni a iniciativas microeconómicas para con el campesinado, quizá resultaría interesante acudir tan sólo de modo testimonial a Corrado Barberis, quien, en 1972 llamaba la atención sobre una serie de medidas adoptadas en Francia que parecían tener un efecto positivo tanto en la reducción del éxodo rural como en la revitalización de la producción agrícola. Por una parte, estaba un mayor interés por parte de los sindicatos hacia un control más efectivo del precio de la tierra; por otra, un más directo y efectivo acuerdo entre cultivadores suprimiendo intermediarios; y, en tercer lugar, una serie de ayudas financieras del estado que redunden en la revalorización de las tierras en proceso de abandono (Van Nieuwenhuijze 1972:19).

Como conclusión y dentro de las posibles soluciones a todo el cúmulo de padecimientos que sufre el mundo campesino y que John Berger denuncia en Pig Earth considero interesante sugerir las palabras ya expresadas por el Papa Juan XXIII en su Encíclica "Mater et Magistra" a las que Faus-Pujol alude en su artículo "Causes of Rural Depopulation":

It is necessary that everyone, particularly the authorities, should strive to develop rural areas and provide them with the basic public services, such as roads, transport, communications, drinking water, housing, medical and pharmaceutical care, elementary, technical and professional education,... When these services, which are necessary to achieve a decent standard of living, are lacking in rural áreas, the economic progress and social development of these areas is either non-existent, or excessively slow, which makes it impossible to reduce the exodus from the land. (1986:19)

\section{BIBLIOGRAFÍA.}

J. Berger, Pig Earth (London 1979) Writers and Readers.

----, Into Their Labours (London 1992) Granta Books.

B. Bogin, "Rural-to-urban migration", Biological aspects of human migration. MascieTaylor \& Lasker eds. (Cambridge Studies in Biological Anthropology 1988) 90-129. Cambridge University Press.

C. Escobedo de Tapia \& J. L. Caramés Lage, El Comentario de Textos AntropológicoLiterarios: Análisis de cinco casos representativos de la novela indo-angla contemporánea (Oviedo 1994) Univ. de Oviedo.

M. C. Faus-Pujol \& A. Higueras-Arnal, Rural depopulation. Commission on population geography. Department of Geography and Spatial Organization (University of Zaragoza 1986) Dip.Gral. de Aragón. Dep. de Cultura y Educación.

N. García Canclini, Las culturas populares en el capitalismo (La Habana 1982) Eds. Casa de las Américas. 
N. Gray, Writers Talking (London 1989) Caliban Books.

E. Iáñez Pareja, El siglo XX: Literatura contemporánea Vol.9 (Barcelona 1995) TesysBosch.

C.G.N. Mascie-Taylor \& G. W. Lasker, Biological aspects of human migration (Cambridge 1988) Studies in Biological Anthropology, Cambridge University Press.

R. Otero Pedrayo, Vivencias, dolor y esperanza de la Emigración Gallega (Santiago de Compostela 1954) Universidad de Santiago de Compostela.

M. Praderie \& M. Passagez, "La mobilité professionnelle en France entre 1959 et 1964", Estudes et conjocture 30 (1966).

J. Riechmann, “John Berger. Contrabando y Resistencia”, Quimera 143 (1995) 12-18.

C. A. O. Van Nieuwenhuijze, Emigration et Agriculture dans le Bassin Mediterranéen (Mouton, The Hague,. Paris 1972) Mediterranean Social Sciences Research Council \& The Institute of Social Studies.

R. Williams, Culture and Society (Harmondsworth, Middlesex 1985) Penguin Books Ltd. 